\title{
An evaluation of organopollutant biodegradation by some selected white rot fungi: an overview
}

\author{
M. Tekere ${ }^{1}$, J. S. Read ${ }^{2} \&$ B. Mattiasson ${ }^{3}$ \\ ${ }^{1}$ Department of Environmental Sciences, \\ School of Agriculture and Environmental Sciences, \\ University of South Africa, South Africa \\ ${ }^{2}$ School of Medicine University of Botswana, Gaborone, Botswana \\ ${ }^{3}$ Department of Biotechnology, Lund University, Sweden
}

\begin{abstract}
An evaluation of indigenous subtropical white rot fungi producing the ligninolytic enzymes laccase and manganese peroxidase only showed that the fungi could degrade synthetic dyes, PAHs and organochlorine pesticides. Biodegradation of the polymeric dye Poly 478 saved as a good screening indicator for organopollutant degradation by the fungi and based on Poly R478, the indigenous fungi; Trametes versicolor, Trametes cingulata, Trametes pocas and unidentified strain DSPM95 were selected as high degraders from 11 screened fungi. PAH biodegradation was evaluated both in batch and continuous culture bioreactors. The degradation of PAHs at an initial concentration of 20 ppm each in static batch cultures over 31 days showed that $+60 \%$ fluorene, $+40 \%$ phenanthrene, $+42 \%$ anthracene and $3-11 \%$ of benzo(a)anthracene and pyrene were degraded. Biodegradation experiments using extra-cellular culture fluid demonstrated that the extracellular enzyme system of the fungi was responsible also for the biodegradation of the PAHs. Studies on PAH biodegradation by the isolate DSPM95 in packed and suspended carrier continuous bioreactors also showed that the fungi could degrade most of the PAH fed continuously at a concentration of $1 \mathrm{ppm}$ over a period of at least 31 days.
\end{abstract}

Keywords: white rot fungi, ligninolytic enzymes, polyaromatic hydrocarbons, synthetic dyes, organochlorine, biodegradation, bioremediation, manganese peroxidase, laccase. 


\section{Introduction}

Polyaromatic hydrocarbons and persistent organochlorine pesticides are some of the major groups of pollutants of concern due to their health effects, such as carcinogenicity, mutagenicity and reproductive effects (Asgher et al. [1]). Microbial biodegradation of organic chemicals is seen as an alternative to physical and chemical decontamination technologies. A large diversity of microorganisms exist in the environment where the pollutants exist and mostly upon exposure to the pollutants the microorganisms can use their metabolic machinery or evolve new metabolic mechanisms to breakdown the pollutants (Peng et al. [2]). Ligninolytic white rot fungi have been shown to degrade a wide range of pollutants including PAHs, synthetic dyes, pesticides and biphenyls (Asgher et al. [1]). Ligninolytic fungi use a system of peroxidases and oxidases to breakdown environmental pollutants. Liginin peroxidase, manganese peroxidase and laccases are the major ligninolytic enzymes and a number of accessory enzymes, such as $\mathrm{H}_{2} \mathrm{O}_{2}$-forming glyoxal oxidase, aryl alcohol oxidase, oxalate producing oxalate decarboxylase (ODC), NAD-dependent formate dehydrogenase (FDH) and P450 monooxygenase (Asgher et al. [1]), have been shown to play important roles in white rot fungi biodegradations. The advantages of a bioremediation system using white rot fungi is that they produce an extracellular, non-specific enzyme system for the degradation of diverse organopollutants, and they require no pre-conditioning to the pollutant for expression of the enzyme system and biodegradation. Although a lot of research progresss has been made in studying fungal applications in bioremediation, a lot still needs to be done in order to identify and quantify all active players from different ecosystems and their biopotentials. This paper gives an overview of the organopollutant biodegradation studies carried out on some white rot fungi from a subtropical forest in Zimbabwe.

\section{Materials and methods}

\subsection{Sampling program}

The fungal strain basidiocarps were collected in August 1995 from Chirinda, a moist evergreen forest, and Chimanimani (miombo woodland) forests in the Eastern Highlands of Zimbabwe. The fungi were growing on dead wood and were the dominant species at the time of collection and are well represented in Zimbabwean woodlands [3]. Pure cultures were obtained and maintained as described in Tekere et al. [4]. The pure isolates obtained were: Trametes elegans (Berk.) Ryvarden, Trametes versicolor (Fr.) Pilat, Trametes cingulata (Berk., Trametes pocas (Berk.) Ryvarden, Isolate DSPM95*, Datronia concentric, Irpex $s p^{*}$, Lentinus velutinus (Fr), Creptidotus mollis (Schaeff.:Fr.) Kummer and Pycnoporus sanguineus (Fr) Murill. With the exception of Irpex $s p^{*}$ and DSPM95*, all the other isolates used were identified using gross and microscopic characteristics (Mswaka [3]). 


\subsection{Laboratory methods}

\subsubsection{Screening for organopollutant biodegradative activity}

Lignin and synthetic dye biodegradations were used as determinants for pollutant biodegradation. Lignin biodegradations were carried out as in the method by Sundman and Nase [5] and described by Tekere et al. [4]. The decolourisation of the synthetic dyes was also determined on agar plates and blue dextran, crystal violet, cresol red and bromophenol blue were used (Sigma Chemical Co., St Louis, MO). The biodegradation experiments were carried out as described in Tekere et al. [4]. The decolourisation of Poly R478 dye was determined in liquid shake cultures using the same medium as described above with the agar excluded. Shake flasks $(500 \mathrm{ml})$, containing $100 \mathrm{ml}$ of medium, were inoculated with four, $6 \mathrm{~mm}$ agar plugs and the flasks were incubated at $30^{\circ} \mathrm{C}$ on a shaker at $100 \mathrm{rpm}$. The decolourisation was determined over a 9 day incubation period by measuring the absorbance ratio 520/350 nm using a Shimadzu Biospec-1601 (Tekere et al. [4]).

Shallow stationery batch cultures were used for the production of the ligninolytic enzymes, lignin peroxidase, manganese peroxidase and laccase using a base medium as proposed by Bonnarme et al. [6]. Culturing and enzyme assays were carried out as described in Tekere et al. [7].

\subsubsection{Polycyclic aromatic hydrocarbon biodegradation}

The biodegradation of representative 2-, 3- and 4- ringed polycyclic aromatic hydrocarbons (PAHs) fluorene, phenanthrene, anthracene, pyrene and benzo(a)anthracene was studied in static batch cultures of the isolates; $P$. chrysosporium, T. versicolor, T. cingulata, T. pocas and DSPM95. Apart from $P$. chrysosporium, the fungal isolates were selected from the indigenous Zimbabwean isolates on the basis of high ligninolytic and high dye decolourisation activities from preliminary screening. Mineral medium as in Tekere et al. [8], was used for culturing the fungi and a mixture of the PAHs at a concentration of $20 \mathrm{mgl}^{-1}$ for each of the PAHs in a $1 \mathrm{ml}$ acetone stock solution was used in $50 \mathrm{ml}$ of the medium in $500 \mathrm{ml}$ shake flasks. The degradation experiments were carried out as described in Tekere et al. [8]. The influence of the addition of glucose oxidase and additional glucose on the biodegradation of the more recalcitrant pyrene and benzo(a)anthracene and the lower molecular weight anthracene was also studied. Glucose oxidase, type V-S from Aspergillus niger, (Sigma Chemical Co) and additional glucose, $5 \mathrm{gl}^{-1}$ were added to cultures after 14 days of incubation. Sampling and PAH extractions and analysis were done as described in Tekere et al. [8]. Effects of addition of glucose oxidase and additional glucose were determined as the hydrogen peroxide - dependant oxidation of phenol red (Tekere et al. [8]).

\subsubsection{PAH biodegradation by fungal extracellular supernatants}

Culture supernatants from 3 week old batch cultures were aseptically separated from the mycelia, centrifuged at $6500 \mathrm{rpm}$, filtered through $0,2 \mu \mathrm{m}$ sterile syringe filters and used for the biodegradation experiments (Tekere et al. [8]). The biodegradations were carried out in triplicate for 6 days for each isolate, 
sampling time and analysis and different experimental conditions and $10 \mathrm{mgl}^{-1}$ of each of the PAHs were used. Samples were frozen at intervals and used for residual PAH analysis. Residual MnP and laccase activity was also determined.

\subsubsection{PAH biodegradation in packed bed and suspended carrier bioreactors}

The white rot fungal isolate DSPM95 was selected for PAH biodegradation studies in packed bed and suspended carrier bioreactor systems on the basis of high dye degradation activity, high ligninolytic activity and high PAH biodegradation activity in batch cultures. A jacketed glass column with a working volume $500 \mathrm{ml}$, filled up with Poraver glass bead carriers which are finely pored glass granules ( 8 - $16 \mathrm{~mm}$ pore size), made from purified recycled glass (Dennert Poraver GMBH, Germany) was used for the packed bed reactor. A jacketed glass column with a working volume of $1000 \mathrm{ml}$, filled with $550 \mathrm{ml}$ kalderns plastic carriers (Anoxa, Lund, Sweden) was used as a suspended carrier bioreactor system. The plastic carriers are cylindrical, with an inner diameter of $8 \mathrm{~mm}$, which is sub-divided into four, and have a rough outside wall allowing for good cell attachment. The assembled reactors were sterilised, inoculated and operated as described in Tekere et al. [9]. Residual PAHs, MnP and laccase activities in the system were followed from the reactor outflow.

\subsubsection{Polycyclic aromatic hydrocarbon extraction and analysis}

Residual PAHs in the samples from both the bioreactors and batch cultures were extracted with hexane and the extraction and analysis of the PAHs is described in Tekere et al. [8] and Tekere et al. [9]. External standards were used for the quantitative determination of the residual PAHs and the detection limit achieved was $0.01 \mathrm{mgl}^{-1}$ for all the compounds. The PAH recovery efficiency for the extraction method was $97-99 \%$ for fluorene, $96 \%$ for phenanthrene, $99 \%$ for anthracene, $94 \%$ for pyrene and $91 \%$ for benzo(a)anthracene. The results are represented as means of the triplicate sample extractions and analyses. The standard deviations were calculated wherever applicable. PAH metabolites were partially identified using GC-MS (Varian Star, 3400, Varian Saturn II) as described in Tekere et al. [8]. A PAH search library (supplied by Varian chromatography systems, Sweden) was used for the qualitative metabolite identification.

\subsubsection{Biodegradation of organochlorines by the fungi}

The organochlorines, lindane, dieldrin, dicofol and endosulfan at a concentration of $20 \mathrm{ppm}$ were incorporated as a mixture into $50 \mathrm{ml}$ growth medium in $500 \mathrm{ml}$ Erlenmeyer flasks. The organochlorines used in these experiments were of technical grade and active ingredient analysis by gas chromatography gave $93 \%$, 95\%, 49\% and 33\% for dicofol, lindane, endosulfan and dieldrin respectively. As controls for non-biological loss, controls in which the pesticides were added to uninoculated contaminated medium were used and both control and test experiments were carried out in the same way as for the fungal PAH degradations described above.

Samples were analysed for residual pesticides using gas chromatography. Sample $(0.5 \mathrm{ml})$ was macerated with $5 \mathrm{ml}$ dichloromethane in a macerating cup 
for $5 \mathrm{~min}$ after which the sample was filtered through sodium sulphate. The dichloromethane was evaporated and the samples were reconstituted in $2 \mathrm{ml}$ hexane for gas chromatography analysis. GC (Varian Star 3400 Cx), fitted with an electron capture detector (ECD) and a capillary column QC 5/BP 10, $25 \mathrm{~cm}$ was used. The detector temperature was set at $300^{\circ} \mathrm{C}$, injector temperature $200^{\circ} \mathrm{C}$ and column temperature was $195^{\circ} \mathrm{C}$. External standards were used in the analyses.

\section{Results and discussions}

As shown in table 1 and fig 1 , all isolates had some lignin and dye decolourisation activity demonstrating potential for pollutant degradation. The isolates DSPM95 and T. versicolor had the highest degradative abilities. No lignin peroxidase activity was detectable in any of the screened isolates used except in the reference strain, $P$. chrysosporium. Even when $\mathrm{Mn}^{2+}$, which is known to reduce LiP titers was excluded (Bonnarme et al. [6]) or when veratryl alcohol was added as an inducer, no LiP could be detected. Laccase and manganese peroxidase activities were detectable in all the tested fungal species and the activity levels were variable within the isolates, with $C$. mollis showing little detectable laccase and manganese peroxidase activities while $T$. versicolor and DSPM95 showed the highest enzyme activities (Tekere et al. [7]).

i) Total PAH degradation in static batch cultures, ND - not detectable,

ii) Total degradation of anthracene, pyrene and benzo(a)anthracene only in static batch cultures at 31 days. The standard deviation was calculated from 3 - 6 samples (Tekere et al. [8]).

Table 1: Decolourisation magnitudes for the dyes and ligninolysis by the fungal isolates. Extent of decolourisation, scale $0-5$ with decolourisation maximum at 5 , as assessed by the area and magnitude of decolourisation. Lignin degradation, $(+),(++),(+++)$ - in order of increasing ligninolysis magnitude.

\begin{tabular}{|c|c|c|c|c|c|}
\hline \multirow{2}{*}{ Isolate } & \multirow{2}{*}{ Lignin } & \multicolumn{5}{|c|}{ Dye degradation } \\
\cline { 3 - 6 } & & C.violet & B.blue & C.red & B.dextran \\
\hline DSPM95 & +++ & 5 & 5 & 5 & 5 \\
\hline T. versicolor & +++ & 5 & 5 & 5 & 5 \\
\hline T. pocas & + & 4 & 3 & 5 & 2 \\
\hline T. cingulata & + & 2 & 5 & 4 & 5 \\
\hline T. elegans & + & 1 & 3 & 3 & 3 \\
\hline D.concentrica & +++ & 2 & 5 & 3 & 3 \\
\hline Irpex spp & ++ & 1 & 5 & 5 & 3 \\
\hline L.velutinus & + & 3 & 4 & 2 & 1 \\
\hline C.mollis & + & 3 & 1 & 3 & 1 \\
\hline P. sanguineus & + & 2 & 3 & 3 & 2 \\
\hline
\end{tabular}

Tekere et al. [4]. 


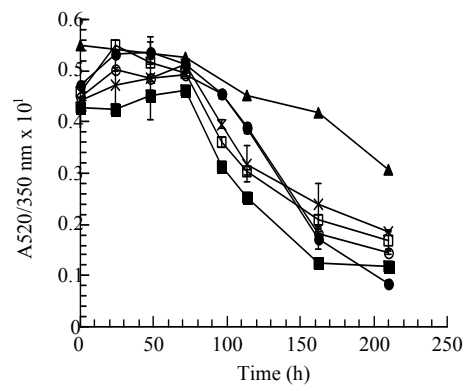

(a)



(b)

Figure 1: Poly R478 decolourisation by white rot fungal isolates in liquid shake cultures $(100 \mathrm{rpm})$ as a function of time. Decolourisation was determined by the absorbance ratio A520/350 $\mathrm{nm}$ : —-T. pocas, —-T. versicolor, $\square-\square$. cingulata, — D. concentrica, Isolate DSPM95 — P. sanguineus, $\multimap-C$. mollis, $\backsim$ L. velutinus, $\longrightarrow$ Irpex spp and $\longrightarrow$ T. elegans (Tekere et al. [4]).

Table 2: $\quad$ Total PAH degraded by the fungal isolates (homogenised whole cultures analysis) as a percentage of the initial concentration. The cultures were incubated at $30^{\circ} \mathrm{C}$ for 31 days.

\begin{tabular}{|c|c|c|c|c|c|}
\hline Isolate & Fluorene & $\begin{array}{l}\text { Phenan- } \\
\text { threne }\end{array}$ & Anthracene & Pyrene & $\begin{array}{c}\text { Benzo(a } \\
\text { anthracene }\end{array}$ \\
\hline \multirow{2}{*}{$\begin{array}{l}\text { P.chryso- } \\
\text { soporium }\end{array}$} & i. $76.0 \pm 6.7$ & $41.7 \pm 1.2$ & $78.0 \pm 10.0$ & $15.0 \pm 5.4$ & $16.0 \pm 2.5$ \\
\hline & & & ii. $90.0 \pm 1.6$ & $13.0 \pm 2.0$ & $15.0 \pm 0.4$ \\
\hline \multirow{2}{*}{$\begin{array}{c}\text { DSPM95 } \\
\text { spp }\end{array}$} & i. $82.0 \pm 3.1$ & $57.0 \pm 2.7$ & $85.0 \pm 10.6$ & $11.0 \pm 0.5$ & $12.0 \pm 2.8$ \\
\hline & & & ii. $98.0 \pm 0.5$ & $52.0 \pm 5.3$ & $33.0 \pm 4.5$ \\
\hline \multirow{2}{*}{$\begin{array}{c}T . \\
\text { versicolor }\end{array}$} & i. $78.0 \pm 5.5$ & $49.0 \pm 7.4$ & $54.0 \pm 7.0$ & ND & $3.0 \pm 0.0$ \\
\hline & & & ii. $82.0 \pm 13.0$ & $9.0 \pm 6.8$ & $30.0 \pm 1.4$ \\
\hline \multirow[t]{2}{*}{ T. pocas } & i. $72.0 \pm 6.3$ & $60.0 \pm 22.0$ & $70.0 \pm 2.6$ & $8.0 \pm 0.6$ & $9.0 \pm 5.4$ \\
\hline & & & ii. $99.0 \pm 0.2$ & $35.0 \pm 2.2$ & $38.0 \pm 2.0$ \\
\hline \multirow[t]{2}{*}{ T. cingulata } & i.63.4 41.6 & $42.0 \pm 3.4$ & $9.0 \pm 2.1$ & $9.0 \pm 2.1$ & $10.0 \pm 4.7$ \\
\hline & & & ii. $99.0 \pm 0.6$ & $40.0 \pm 10$ & $23.0 \pm 5.6$ \\
\hline
\end{tabular}

The isolated fungi had high degradative activity for flourene, anthracene and phenanthrene and degradation was low for high molecular weight PAHs, pyrene and benzo(a) anthracene. PAH adsorption to fungal mycelium occurred and as shown in table 3 , high pyrene and benzo(a)anthracene remained associated with mycelium at the end of the culturing period.

PAH biodegradation in fungal extracellular fluids under different experimental treatments showed that fungal secretions in the extracellular media was responsible for the biodegradation and addition of $\mathrm{H}_{2} \mathrm{O}_{2} / \mathrm{Mn}^{2+}$ and/ ABTS caused an increase in the degradation of the PAHs in most cases though the effects were variable for different fungi. Anthracene was most degraded in 
Table 3: $\quad$ The percentage PAH associated with the mycelia of the fungal strains. This percentage PAH sorption was calculated from the difference in the PAH degraded as analysed from the culture supernatant extractions and the PAH degraded as analysed from the homogenised whole culture extractions.

\begin{tabular}{|c|c|c|c|c|c|}
\hline \multirow{2}{*}{ Isolate } & \multicolumn{5}{|c|}{ \% PAH associated with the mycelia } \\
\cline { 2 - 6 } & Fluorene & Phenanthrene & Anthracene & Pyrene & $\begin{array}{c}\text { Benzo(a) } \\
\text { anthracene }\end{array}$ \\
\hline P. chrysosporium & $10.0 \pm 2.0$ & $7.0 \pm 0.0$ & $16.0 \pm 5.0$ & $54.0 \pm 6.4$ & $52.0 \pm 10.0$ \\
\hline DSPM95 & $6.0 \pm 0.8$ & $22.0 \pm 3.5$ & $8.0 \pm 3.4$ & $40.0 \pm 11.0$ & $53.0 \pm 5.5$ \\
\hline T. versicolor & $5.0 \pm 0.15$ & $25.0 \pm 5.0$ & $26.0 \pm 8.8$ & $36.0 \pm 16$ & $32.0 \pm 23$ \\
\hline T. pocas & $10.0 \pm 6.0$ & $33.0 \pm 5.1$ & $26.0 \pm 0.5$ & $54.0 \pm 0.5$ & $33.0 \pm 11.0$ \\
\hline T. cingulata & $4.2 \pm 2.0$ & $22.0 \pm 10.0$ & $10.0 \pm 3.0$ & $36.0 \pm 2.0$ & $46.0 \pm 8.4$ \\
\hline
\end{tabular}

Tekere et al. [8]

Table 4: $\quad$ PAH biodegradation in extracellular fluids under different experimental treatments, incubated at $30^{\circ} \mathrm{C}$ for 6 days.

\begin{tabular}{|c|c|c|c|c|c|c|}
\hline \multirow[t]{2}{*}{ Isolate } & \multirow[t]{2}{*}{ Culture treatment } & \multicolumn{5}{|c|}{$\%$ PAH degraded } \\
\hline & & $\begin{array}{c}\text { Fluoren } \\
\mathrm{e}\end{array}$ & $\begin{array}{l}\text { Phenanth } \\
\text { rene }\end{array}$ & $\begin{array}{l}\text { Anthra } \\
\text { cene }\end{array}$ & Pyrene & $\begin{array}{c}\text { Benzo(a) } \\
\text { nthracen } \\
\mathrm{e}\end{array}$ \\
\hline \multirow{3}{*}{$\begin{array}{l}\text { P.chryso- } \\
\text { soporium }\end{array}$} & Culture fluid & 28.0 & 15.0 & 17.5 & 8.0 & 4.9 \\
\hline & $+\mathrm{H}_{2} \mathrm{O}_{2} / \mathrm{Mn}^{2+}$ & 69.0 & 32.5 & 66.5 & 36.0 & 22.0 \\
\hline & $+\mathrm{H}_{2} \mathrm{O} 2 / \mathrm{Mn}^{2+} / \mathrm{ABTS}$ & 41.0 & 37.5 & 16.0 & 29.0 & 23.0 \\
\hline \multirow[t]{3}{*}{ DSPM95 spp } & Culture fluid & 56.0 & 29.5 & 55.0 & 15.0 & 25.0 \\
\hline & $+\mathrm{H}_{2} \mathrm{O}_{2} / \mathrm{Mn}^{2+}$ & 72.0 & 35.0 & 79.0 & 22.0 & 53.0 \\
\hline & $+\mathrm{H}_{2} \mathrm{O} 2 / \mathrm{Mn}^{2+} / \mathrm{ABTS}$ & 71.0 & 43.0 & 63.0 & 37.5 & 32.0 \\
\hline \multirow[t]{3}{*}{ T. versicolor } & Culture fluid & 42.0 & 19.5 & 23.5 & 7.0 & 8.5 \\
\hline & $+\mathrm{H}_{2} \mathrm{O}_{2} / \mathrm{Mn}^{2+}$ & 77.5 & 37.5 & 43.0 & 20.0 & 52.5 \\
\hline & $+\mathrm{H}_{2} \mathrm{O}_{2} / \mathrm{Mn}^{2+} / \mathrm{ABTS}$ & 70.0 & 39.0 & 30.5 & 31.5 & 58.0 \\
\hline \multirow[t]{3}{*}{ T. pocas } & Culture fluid & 38.5 & 23.0 & 29.5 & 17.0 & 18.2 \\
\hline & $+\mathrm{H}_{2} \mathrm{O}_{2} / \mathrm{Mn}^{2+}$ & 64.0 & 38.0 & 77.0 & 19.5 & 30.5 \\
\hline & $+\mathrm{H}_{2} \mathrm{O}_{2} / \mathrm{Mn}^{2+} / \mathrm{ABTS}$ & 67.0 & 61.0 & 32.0 & 33.0 & 38.0 \\
\hline \multirow[t]{3}{*}{ T.cingulata } & Culture fluid & 24.0 & 19.5 & 22.0 & 12.0 & 13.0 \\
\hline & $+\mathrm{H}_{2} \mathrm{O}_{2} / \mathrm{Mn}^{2+}$ & 71.0 & 32.5 & 32.0 & 19.5 & 18.0 \\
\hline & $+\mathrm{H}_{2} \mathrm{O}_{2} \mathrm{Mn}^{2+} / \mathrm{ABTS}$ & 66.5 & 35.0 & 22.5 & 15.5 & 21.0 \\
\hline
\end{tabular}

conditions with $\mathrm{H}_{2} \mathrm{O}_{2} / \mathrm{Mn}^{2+}$ while phenanthrene was most degraded under $\mathrm{H}_{2} \mathrm{O}_{2}$ / $\mathrm{Mn}^{2+}$ and/ ABTS conditions. In controls only $20.5 \%$ and $9.2 \%$ of fluorene and phenanthrene respectively was not recovered, thus attributed to abiotic loss, all of the other PAHs was recovered from the controls. Determination of residual enzyme activity in the extracellular fluid of the fungal cultures showed that the enzymes remained relatively stable over an 8 -day incubation period at $92 \%$ for $\mathrm{MnP}$ and $88 \%$ for laccase.

Metabolic intermediates occurred during PAH biodegradation and several metabolic peaks were evident during PAH analysis. Comparison of the pattern of PAH degradation in $P$. chrysosporium and $T$. pocas, $T$. cingulata, $T$. 
versicolor and DSPM95 based on GC-MS pattern showed that there was more metabolite breakdown by the other fungi than $P$. chrysosporium at 31 days. As an example Fig 2a and b shows the GC-MS pattern for $T$. pocas and $P$. chrysosporium respectively at 31 day incubation time. This shows that different fungi should be explored in order to come up with the best candidates for complete degradation of the PAHs.

Evaluation of PAH biodegradation in both the packed bed and suspended carrier bioreactors showed that high degradation and enzyme activities could be achieved with immobilised fungi. Since fungi are slow growing microorganisms and prefer growing attached to surfaces, retaining them in immobilised bioreactors is an advantage. Fig 3a and a show the PAH biodegradation and enzyme activities for DSPM95 in the packed bed bioreactor. A similar profile was obtained for the suspended carrier bioreactor as presented in Tekere et al. [9]. Both reactor configurations could be used in bioremediation of polluted waste effluent, the suspended carrier however offers the advantage of good fluid mixing and no clogging with long term operation like what happens with the void pore spaces in packed bed reactors.

Control reactor studies showed that PAH recovery was low in the initial stages of the reactor operation but PAH recovery at a fixed flow rate increased with time while PAH recovery at different flow rates, increased with increased flow rate. For example the PAH recovered increased with time from $30.0 \%$ to

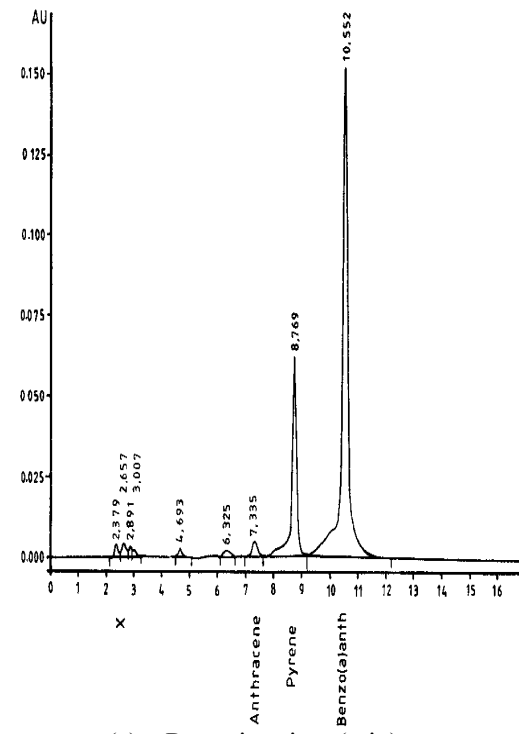

(a) Retention time ( $\mathrm{min})$

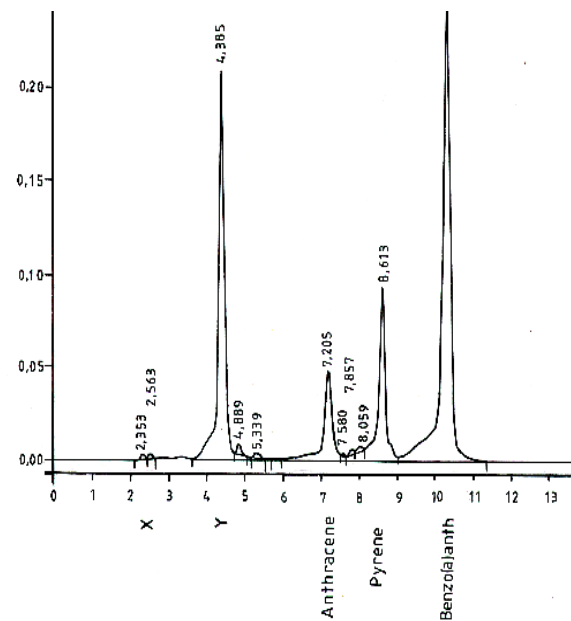

(b) Retention time (min)

Figure 2: Chromatograms for the biodegradation of anthracene, pyrene and benzo(a) anthracene in 31 day old cultures of (a) T. pocas and (b) $P$. chrysosporium with glucose and glucose oxidase added. $\mathrm{x}$ and $\mathrm{y}$ are metabolic peaks (Tekere et al. [8]). 


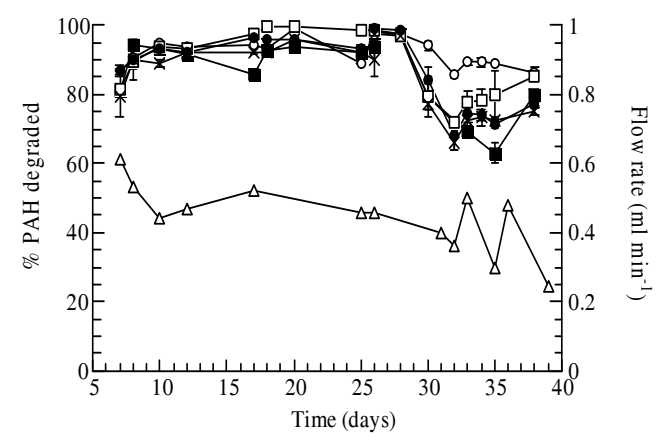

(a)

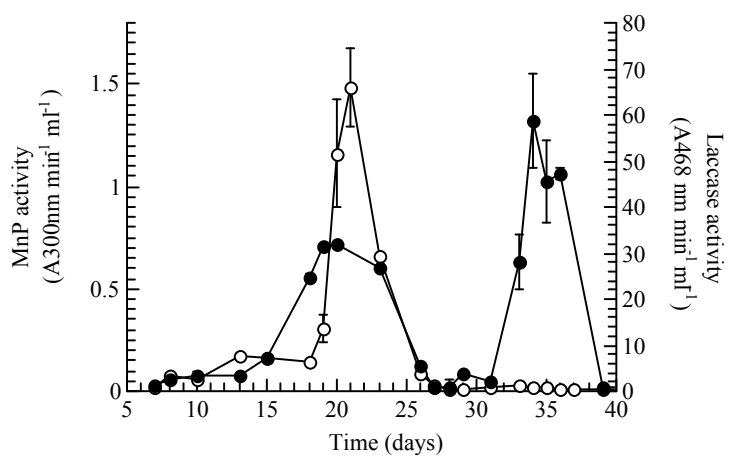

(b)

Figure 3: Biodegradation of the PAHs by DSPM95 and changes in enzyme activities and medium flow rates in the packed bed bioreactor system. (a) - $\quad$ fluorene, $\square$ phenanthrene, $\square \square$ anthracene, $\longrightarrow$ pyrene, $\longrightarrow$ benzo(a) anthracene and flow rate. (b) — $\longrightarrow$ nnP and $\longrightarrow$ laccase activity. The reactor operation conditions were: (i) day 7-13 basal feed medium with no glucose was fed; (ii) day 13-29 medium with $2 \mathrm{~g}$ glucose, $25 \mathrm{ppm} \mathrm{Mn}^{2+}$ and $0.66 \mathrm{~g} \mathrm{~N}$ (a medium optimum for enzyme production for the isolate DSPM95); (iii) day 29-36 medium with no glucose, $25 \mathrm{ppm} \mathrm{Mn}^{2+}$ and $0.66 \mathrm{~g} \mathrm{~N}$; (iv) day 3640 medium with $2 \mathrm{~g}$ glucose, $25 \mathrm{ppm} \mathrm{Mn}^{2+}$ and $0.66 \mathrm{~g} \mathrm{~N}$ (Tekere et al. [9]).

almost $80.0 \%$ for the packed bed reactor at a flow rate of $0.5 \mathrm{ml} \mathrm{min}{ }^{-1}$. This could be attributed to the saturation of the absorption capacity of the carriers. Determination of the PAH adsorbed to the carrier material revealed that around $2.0 \%$ flourene, phenanthrene and anthracene, and $21.0 \%$ and $13.0 \%$ pyrene and benzo(a)anthracene respectively could be recovered from the carriers. Airlift of the PAHs from the control reactors also occurred. PAH lost from the medium in 
Table 5: Percentage PAH degraded at different flow rates in the suspended carrier bioreactor.

\begin{tabular}{|c|c|c|c|c|c|}
\hline \multirow{2}{*}{$\begin{array}{c}\text { Flow rate } \\
\left(\mathrm{mlmin}^{-1}\right)\end{array}$} & \multicolumn{5}{|c|}{ \% PAH degraded } \\
\cline { 2 - 6 } & Fluorene & Phenathrene & Anthracene & Pyrene & $\begin{array}{c}\text { Benzo(a) } \\
\text { anthracene }\end{array}$ \\
\hline 0.5 & $94.7 \pm 0.7$ & $94.0 \pm 1.0$ & $97.0 \pm 0.1$ & $92.0 \pm 2.0$ & $91.0 \pm 1.0$ \\
\hline 0.82 & $95.6 \pm 2.0$ & $93.1 \pm 0.9$ & $96.9 \pm 0.9$ & $92.4 \pm 3.1$ & $92.0 \pm 0.5$ \\
\hline 1.1 & $75.4 \pm 6.0$ & $83.9 \pm 1.5$ & $89.5 \pm 3.2$ & $85.0 \pm 11$ & $85.1 \pm 3.0$ \\
\hline
\end{tabular}

Results are given as \% mean recovery from at least 2 to 4 samples with standard deviation. Tekere et al. [9].

Table 6: Lindane, dicofol, endosulfan and dieldrin degradation in static batch cultures of some white rot fungi in 31 days.

\begin{tabular}{|c|c|c|c|c|}
\hline \multirow{2}{*}{ Isolate } & \multicolumn{4}{|c|}{ \% Pesticide degraded } \\
\cline { 2 - 5 } & Lindane & Dicofol & Endosulfan & Dieldrin \\
\hline DSPM95 & 84.0 & 95.0 & 97.0 & 98.0 \\
\hline T. pocas & 99.0 & 97.0 & 98.0 & 98.0 \\
\hline P. sanguineus & 99.0 & 99.0 & 99.0 & 98.0 \\
\hline D.concentrica & 93.0 & 99.0 & 98.0 & 98.0 \\
\hline T. versicolor & 61.0 & ND & 50.0 & 30.0 \\
\hline T. cingulata & 62.0 & ND & 50.0 & 99.0 \\
\hline
\end{tabular}

ND - pesticide degradation not well determined.

the suspended carrier bioreactors through airlift at a airflow rate of $1.41 \mathrm{~min}^{-1}$ after $48 \mathrm{~h}$ was $43 \%, 36 \%, 32 \%, 27 \%$ and $26 \%$ for flourene, phenanthrene, anthracene, pyrene and benzo(a)anthracene respectively. However it should be noted that in inoculated reactors adsorbed PAHs will be degraded and the growing biomass can contribute to reduction of the PAHs lost though aeration.

The degradation of the organochlorines; lindane, dicofol, endosulfan and dieldrin by the fungal isolates is shown in Table 6 . With the exception of the isolates $T$. versicolor and $T$. cingulata all the other fungal isolates consistently degraded the pesticides dicofol, endosulfan and dieldrin by above $95.0 \%$ of the initial parent compounds showing their potential in pesticide bioremediation. Further studies on lindane degradation are reported in Tekere et al. [10].

\section{Conclusions}

The ability of the white rot fungi to degrade a wide range of pollutants means that they can be best for application in the biodegradation of mixed pollutants in soil and wastewater, where a considerable diversity of organopollutants is often found. The white rot fungi reported here can be explored further for bioremediation of synthetic dyes, PAHs and organochlorine pesticides among other pollutants thus offering a solution in the clean-up of the environments contaminated by these pollutants. Continuous screening of mainly those basidiomycetes white rot fungi which have not yet been studied can yield isolates which can produce not only high ligninolytic enzyme levels but can also cause complete pollutant degradation. This study also shows that some of the 
studied white rot fungi with only $\mathrm{MnP}$ and laccase activities actually have higher biodegradation capabilities than the model fungus $P$. chrysosporium which is a $\mathrm{LiP}$ and MnP producer. While the model fungus $P$. chrysosporium showed the accumulation of anthraquinone, the degradative pathways for PAHs by selected sub - tropical white rot fungi showed further metabolism of the intermediate metabolites such as anthraquinone. The white rot fungi can also be used successfully in continuous packed bed and suspended carrier bioreactors to achieve high and prolonged pollutant degradation.

\section{References}

[1] Asgher, M., Bhatti, H.N., Ashraf, M. \& Legge, R.L. Recent developments in biodegradation of industrial pollutants by white rot fungi and their enzyme system. Biodegradation, 19, pp. 771-783, 2008.

[2] Peng, R., Xiong, A., Xue, Y., Fu, X., Gao, F., Zhao, W. \& Tian, Y. Microbial degradation of polyaromatic hydrocarbons. FEMS Microbiology Reviews 32, pp. 927-955, 2008.

[3] Mswaka, A.Y. Studies on Trametes occurring in indigenous forests of Zimbabwe. PhD Thesis, Cranfied University, UK, 1995.

[4] Tekere, M., Mswaka, A.Y., Zvauya, R. \& Read, J.S. Growth, dye degradation and ligninolytic activity studies on Zimbabwean white rot fungi. Enzyme and Microbial Technology, 28, pp. 420 - 426, 2001.

[5] Sundman, V. \& Nase, L. A simple plate test for direct visualisation of biological lignin degradation. Paper and Timber, 2, pp. 67 - 71, 1971.

[6] Bonnarme, P., Perez, J. \& Jeffries, T.W. Regulation of ligninase production in white rot fungi. In Leatham, G.F. and Himmel, M.E. (Eds.), Enzyme in Biomass Conversion, ACS symposium Series 460, ACS, Washington, D.C. pp. $200-206,1991$.

[7] Tekere, M., Zvauya, R. \& Read, J.S. Ligninolytic enzyme production from selected sub - tropical white rot fungi under different culture conditions. Journal of Basic Microbiology, 41, pp. 97 - 111, 2001.

[8] Tekere, M., Read, J.S. \& Mattiasson, B. Polycyclic aromatic hydrocarbon biodegradation in extracellular fluids and static batch cultures of selected Sub - Tropical white rot fungi. Journal of Biotechnology, 115, pp. 367$377,2005$.

[9] Tekere, M. Read, J.S. \& Mattiasson, B. Polycyclic aromatic hydrocarbon Biodegradation by a sub - tropical white rot fungus in packed-bed and suspended carrier bioreactor systems. Environmental Technology, 28 pp $683-691,2007$.

[10] Tekere, M., Ncube, I., Read, J.S. \& Zvauya, R. Biodegradation of the organochlorine pesticide, lindane by a tropical white rot fungus in batch and packed bed bioreactor systems. Environmental Technology, 23, pp. 199-206, 2002. 